\title{
Molecular Characterization of Staphylococcus aureus Isolates Obtained from Hemodialyzed Patients at the Hospital de Clínicas of Paraguay: A pilot study
}

Rubén Arturo Silvero-Isidre,' Fátima Rodríguez-Acosta, ' César Rodrigo Cristaldo-Vargas,' Genaro Américo Velázquez-Romero,' José Félix Plans-Perrota,' Rosa María Guillén-Fretes.'

\begin{abstract}
Background: Patients undergoing hemodialysis are susceptible to the nasal carriage of Staphylococcus aureus, increasing the risk of developing infections associated with higher morbidity and mortality. The objective of this study was to describe the frequency of $\mathrm{S}$. aureus carriage in hemodialysis patients and to perform molecular analysis of isolates by applying multiple-locus variable analysis. Methods: We conducted a descriptive cross sectional study with non-probabilistic sampling that included 28 hemodialysis patients attending the Nephrology Department of Hospital de Clínicas in Asunción, Paraguay. We obtained clinical data from medical records and interviews with patients. Nasal swabs were collected and analyzed by microbiological and molecular methods. Results: The frequency of $\mathrm{S}$. aureus carriage was $50 \%(14 / 28), 93 \%$ of which $(13 / 14)$ were methicillin resistant, $57 \%(6 / 14)$ were gentamicin resistant and $36 \%(5 / 14)$ were resistant to more than 4 antibiotic classes. S. aureus carriers showed higher frequency of rhinitis $(p=0.02$ odds ratio [OR] $=6.6$ ( 1.2 34.4)). Seven methicillin-resistant $S$. aureus isolates had been analyzed by multiple-locus variable analysis, two of them showed identical pattern bands. Conclusion: We found a high frequency of methicillin-resistant Staphylococcus aureus colonization and the presence of two isolates with identical profile in the multiple-locus variable analysis indicating the possibility of transmission between patients.
\end{abstract}

Keywords: Staphylococcus aureus, Hemodialysis, Antibiotic resistance, bacterial typing (Source: MeSH-NLM).

About the Author: Arturo Silvero Isidre is a final-year medical student of a six-year program at the Universidad Nacional de Asunción, Paraguay. He is editor of a scientific journal called CIMEL and a member of a scientific society of medical students (SOCIEM-UNA) in Paraguay.

\section{Introduction}

S. aureus is a common pathogen causing bloodstream infections in the hospital environment. The possibility of nasopharyngeal colonization increases the risk of endogenous infections, and is linked to the $80 \%$ of cases of invasive S. aureus infections.' This microorganism was frequently reported as a pathogen in patients undergoing dialysis and kidney transplantation. ${ }^{2}$ These individuals have risk factors for colonization and infection with multidrug-resistant $\mathrm{S}$. aureus because they are exposed to frequent and prolonged use of antimicrobials. ${ }^{3}$ Furthermore, the need to use invasive devices such as catheters for venous access are associated with a high risk of bloodstream infections. ${ }^{4.5}$ In Paraguay the data about this pathogen in hemodialysis patients is scarce and is limited to local studies that have not been published. Other risk factors for methicillin resistant S. aureus (MRSA) carriage are age . 75 years, prolonged hospitalization, history of repeated administration of antibiotics, type of vascular access, the frequency of hospitalization, immunosuppressive therapy, the use of heparin in the middle of treatment, iron overload, lack of hygiene, comorbidities and proximity to other people carrying $S$. aureus ${ }^{6,7}$

Despite the great technological advances, the mortality rates in hemodialyzed (HD) patients remain unsatisfactorily high. Along with cardiovascular disease, infections are the leading causes of morbidity, hospitalization and mortality in this population.
The annual mortality rate for sepsis is 100 to 300 times higher in patients with end stage renal disease than in the general population, and there is evidence of the association between nasal carriage of MRSA and poor clinical outcome in HD outpatients. ${ }^{6-8}$

Molecular typing has been used to perform epidemiological studies at the global level. In a specific environment over a short period of time, microorganism typing techniques are used to study nosocomial outbreaks, local transmission, and the relationship between carriage and infection in patients. ${ }^{9}$

The multiple-locus variable number of tandem repeat analysis (MLVA) method can be used for the analysis of genetic variability of $\mathrm{S}$. aureus isolates because it has a high discriminatory power for the characterization of bacterial isolates, and it is based on the variation of 7 different loci. The analysis generates multiple PCR products that differs in size for each allele and produces a pattern of 7 bands like a code bar. Isolates that are genetically distant present differences in their profile of bands and those that are identical share the same pattern of bands., ${ }^{3,10}$ The objective of this pilot study was to describe the frequency of $S$. aureus carriage in hemodialyzed patients attending the nephrology department of the Hospital de Clínicas, and perform the clustering analysis of isolates by the MLVA molecular technique. 
Table 1. Clinical outcomes when comparing the interventions.

\begin{tabular}{lcc}
\hline & Sensible \% (n) & Resistance \% (n) \\
\hline Oxacillin & $7 \%(1)$ & $93 \%(13)$ \\
\hline Erythromycin & $21 \%(3)$ & $79 \%(11)$ \\
\hline Ciprofloxacin & $29 \%(4)$ & $71 \%(10)$ \\
\hline Clindamycin & $36 \%(5)$ & $64 \%(9)$ \\
\hline Centamicin & $43 \%(6)$ & $57 \%(8)$
\end{tabular}

\section{Patients and Methods Study Design}

A descriptive cross-sectional pilot study with a non-probabilistic sampling of consecutive cases was performed on 0ctober 2013, including 28 patients undergoing hemodialysis in the Nephrology Department of Hospital de Clínicas from Paraguay. The study was approved by the Facultad de Ciencias Médicas Ethics Committee of the Universidad Nacional de Asunción. Patients received information about the study and those who agreed to participate voluntarily signed an informed consent form. For the data collection process, we interviewed patients and revised the medical records. Two patients with missing data concerning this study were excluded. The data collected included the patient's age, sex, years undergoing hemodialysis, white blood cell count, differential count of lymphocytes, neutrophils and eosinophils expressed as number of cells/mm3, day and turn of hemodialysis, use of antibiotics over the last six months, the use of an invasive device, and history of rhinitis.

\section{Collection of samples}

We collected the nasal swab of dialysis patients and preserved them in Stuart medium for transportation to the microbiology laboratory. The samples were cultured on blood agar, chocolate agar, mannitol agar and incubated at $37^{\circ} \mathrm{C}$ for 24 hours. The identification of S. aureus (STAPH-PLUS Pastorex, USA) included the following tests: Gram staining and biochemical test of catalase, DNase, and the agglutination test.

\section{Susceptibility}

We have implemented the disk diffusion Kirby-Bauer method according to the Clinical and Laboratory Standards Institute (CLSI) in order to identify the antimicrobial susceptibility. We evaluated the susceptibility to oxacillin, ciprofloxacin, erythromycin, ampicillin-sulbactam, clindamycin, and gentamicin as well. The susceptibility to oxacillin was tested through the use of a cefoxitin disk considered as a breakpoint for resistance if the growing inhibition zone of diameter, $21 \mathrm{~mm}$. Susceptibility to vancomycin was not evaluated due to lack of access to methods to determine the minimum inhibitory concentration. Intermediate susceptibility results were considered resistant.

\section{Molecular Methods}

We confirmed isolates as $S$. aureus molecularly through the amplification of a specific 16 S rRNA gene, using the protocol and oligonucleotides described by Manfredi et al. 201011. We have extracted DNA from isolated colonies on blood agar by using the commercial kit Wizard Genomic (Promega, USA) following the manufacturer instructions. DNA quantification was performed using the UV spectrophotometer Biowave DNA (Cambridge, UK). The analysis of the genetic variability of $\mathrm{S}$. aureus isolates was carried out by the MLVA technique. This technique involves amplifying seven loci using oligonucleotides and was previously described by Sabat and collaborators. ${ }^{12}$ The strain ATCC $\circledast 29213$, negative mecA and producing weak

-lactamase was included as a control in each MLVA assay. We separated the PCR products on $7.5 \%$ polyacrylamide gels and performed silver staining following the protocol described by Sambrook et al. ${ }^{13}$ We captured digital images with the Kodak Digital Science team CD120 system (Kodak, NYC, United States). We used TreeCon 1.3b software (Chent University, Gante, Belgium) for the analysis of the band patterns generated and the design of dendrograms.

\section{Statistical issues}

We have compared continuous variables such as: patients age, years undergoing hemodialysis, white blood cell count, differential count of lymphocytes, neutrophils, and eosinophils (expressed as number of cells/mm3) between patients with MRSA carriage versus patients without MRSA carriage through the Student's t-test; and dichotomous variables as well such as: patients sex, day and turn of hemodialysis, use of antibiotics over the last six months, the use of an invasive devi$c e$, and history of rhinitis were analyzed using the chi-square test. Statistical significance was defined as a $p$ value of $p<0.05$ using SPSS 15.0 software (IBM, NYC, United States) for statistical analysis.

Table 2. Resistance profile of Staphylococcus aureus isolates.

\begin{tabular}{ll}
\hline & Percentage (n) \\
\hline More than 1 antibiotic classes & $93 \%(13)$ \\
\hline More than 2 antibiotic classes & $86 \%(12)$ \\
\hline More than 3 antibiotic classes & $50 \%(7)$ \\
\hline More than 4 antibiotic classes & $36 \%(5)$ \\
\hline
\end{tabular}




\section{Results}

Among the 28 HD patients from the Nephrology Department of Hospital de Clínicas who were part of this study, 50\% (14/28) of them were carriers of $\mathrm{S}$. aureus. Antibiotic susceptibility testing identified $92.9 \%(13 / 14)$ of MRSA carriage. Isolates were also resistant to other antibiotics, especially erythromycin and ampicillin-sulbactam, both with $78.6 \%(11 / 14) .42 .9 \%(6 / 14)$ of the isolates were sensible to Gentamicin (Table 1). Isolates that showed resistance to more than 4 families of antibiotics were considered multiresistant and included $35.7 \%(5 / 14)$ of MRSA isolates (Table 2).

The analysis of the demographic and clinical data of the hemodialyzed patients classified as: S. aureus carriers and non-carriers showed that only the differences registered in rhinitis $\mathrm{OR}=6.6(95 \% \mathrm{Cl}: 1.2-34.4, \mathrm{p}=0.022)$ were statistically significant (p<0.05) (Table 3).

Table 3. Demographics of Hemodialyzed Patients
All the isolates identified as S.aureus by biochemical methods $(n=14)$ were confirmed by the detection of 165 rRNA gene by PCR amplification, with a $100 \%$ agreement between phenotypic and genotypic methods. PCR amplification of the 7 loci included in the MLVA method were optimal for cluster analysis in 7 of the 14 MRSA isolates in the study and it gave us the chance to determine the genetic variability (Table 4). The bioinformatic analysis of the band profiles obtained in these isolates generated a dendrogram which is showed in Figure 1. This graphic display has the form of a tree, in which the distance between braches indicates the genetic difference. It can be observed that isolates identified as CR-4 and CR- 5 show the same band profile and are clustered together in one branch at the same distance, whereas isolates CR-10 and CR-12, and CR-9 and CR14 as well, show similar but not identical band profiles and are clustered in groups with branches relatively close to each other (Figure 1).

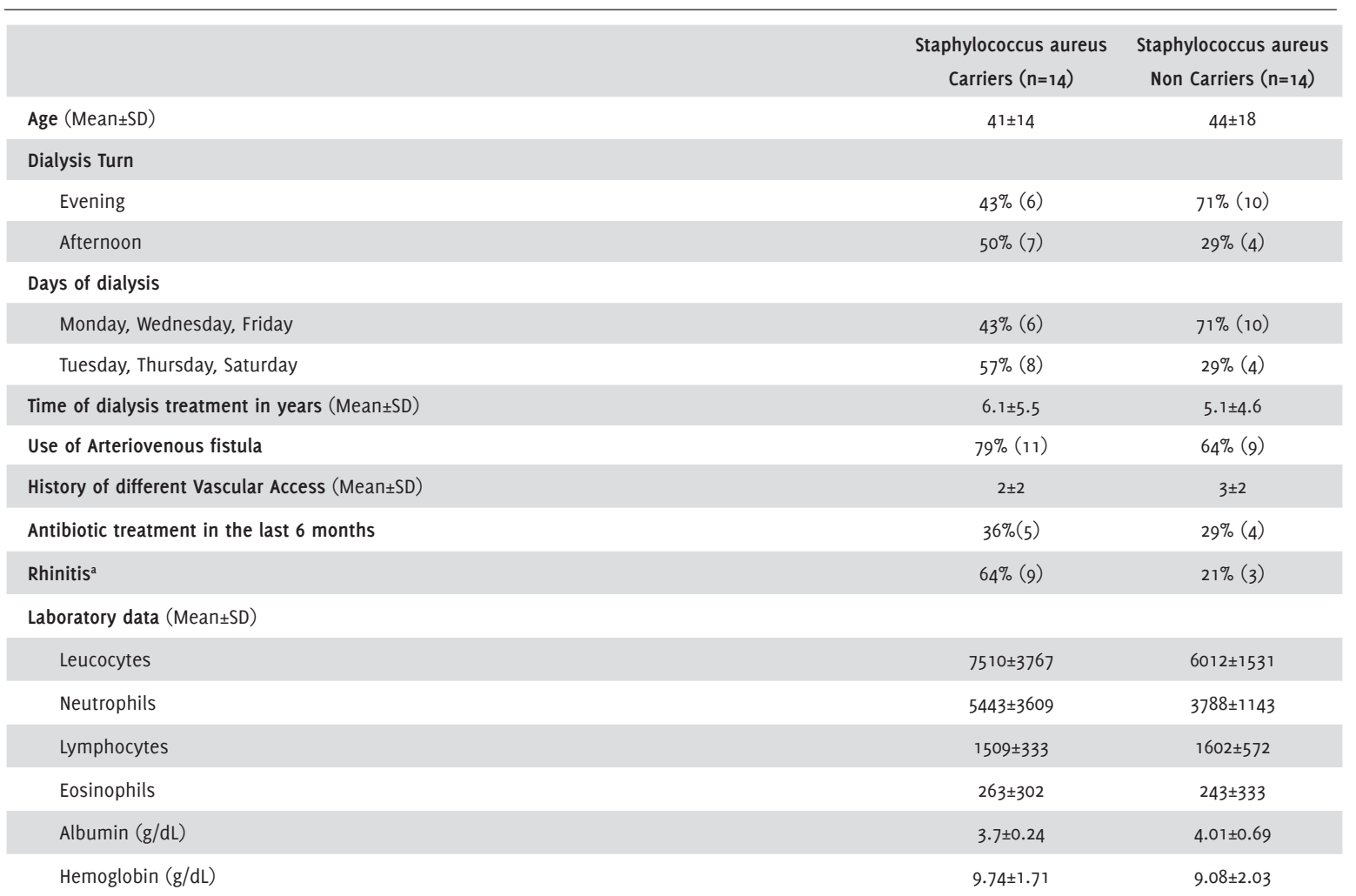

a Difference was statistically significant.

\section{Discussion}

The results showed an alarming frequency of Staphylococcus aureus carriage in hemodialyzed patients (50\%), considering that carrying this organism is an important cause of morbidity and mortality in patients who receive hemodialysis. This study portrayed a high prevalence of nasal $\mathrm{S}$. aureus carriage compared with others studies ${ }^{14,15}$ that showed between $5-13 \%$ of $S$. aureus carriage. ${ }^{14,15}$ However, two studies demonstrated similar carriage rates of $\mathrm{S}$. aureus in hemodialysis patients including Verhoeven et al $(58 \%)^{16}$ and Price et al $(49 \%)$. $^{17}$ The risk of infections in nasal carriers of this microorganism is real and well defined. ${ }^{18}$ It is important to point out that the small number of patients included in this study is a limitation and that the results cannot be extrapolated to other hemodialysis services. However, the data regarding this topic is the first published in Paraguay and further research or systematic studies could be done throughout the country in the future.

There are several studies on MRSA carriage, which reported variable data. One meta-analysis by Zacharioudakis et al.19 found $6 \%$ of carriage; the frequency in this study was much higher $(46.4 \%)$. MRSA carriers had an increased risk of mortality in all diseases. This is attributable to the characteristics of the patients that are carriers of MRSA, who exhibit an impaired immune response, and as a consequence lead to an increased 
Table 4. Phenotypical and Cenotypical Characteristics of Isolates Typed by MLVA.

\begin{tabular}{|c|c|c|c|c|c|c|c|c|c|c|c|}
\hline 4 & $M$ & 53 & Afternoon & CR-4 & $R$ & $R$ & $R$ & $R$ & $R$ & $R$ & A \\
\hline 9 & $\mathrm{~F}$ & 57 & Evening & CR-9 & $R$ & $R$ & $R$ & 1 & $R$ & 1 & E \\
\hline 10 & M & 58 & Afternoon & CR-10 & 1 & 1 & 1 & $R$ & S & S & B \\
\hline 14 & $\mathrm{~F}$ & 32 & Evening & CR-14 & $R$ & $S$ & 1 & S & 1 & I & $\mathrm{F}$ \\
\hline 17 & M & 20 & Evening & CR-17 & $\mathrm{R}$ & $R$ & $\mathrm{R}$ & R & $R$ & $R$ & D \\
\hline
\end{tabular}

MLVA: Multiple Locus Variable Number of Tandem Repeat Analysis; HD: Hemodialysis; CR: Sample code; M=Male; F=Female; R=Resistant; I=Intermediate; S=Sensitive; O=Methicillin; E=Erythromycin; A=Ampicillin-sulbactam; L=Clindamycin; C=Centamicin; C=Ciprofloxacin.

risk of infections. ${ }^{8}$ Risk factors predispose not only for MRSA carriage, but also for multidrug-resistant gram negative baciIli (GNB) and vancomycin-resistant enterococci (VRE). ${ }^{20}$ There is the possibility of vancomycin resistance in MRSA, through the transmission of the vanA gene, as occurred in neighboring countries. ${ }^{21}$ In accordance with the high level of MRSA carriage, $32 \%$ of the isolates in the study were resistant to all antibiotics used in the Kirby-Bauer test, especially erythromycin (79\%). Gentamicin was the antibiotic which had the most effective action in-vitro ( $43 \%$ sensitivity), but the resistance rates were high as well. This study showed similar rates of resistance compared to other countries within the region. ${ }^{22}$ The high rates of resistance to antibiotics by MRSA display the capability of the pathogen to carry more resistance genes than MSSA.

It is important to mention that we did not test the susceptibility to vancomycin in this study; this would be relevant for the epidemiological surveillance and behavior of strains in the hospital environment. In our country, VISA (vancomycin-intermediate S. aureus) or hVISA (heterogeneous vancomycin-intermediate $S$. aureus) have not been reported yet. This issue could be associated with the restricted access to an automated method to test vancomycin resistance by the minimum inhibitory concentration (MIC) method that is not available in all microbiology laboratories.

By using the MLVA technique, we identified 2 isolates that showed identical band profiles. These isolates also shared the same antimicrobial spectrum (CR-4, CR-5), showing the possibility that they correspond to the same clone. This should be analyzed by the combination of other molecular methods such as field gel electrophoresis (PFGE), multilocus sequencing typing and spa typing.

We discarded the possibility of epidemiological outbreaks due to the low number of isolates having identical MLVA profiles, but we have not excluded any risk of transmission between patients in the service. Internal transmission taking place in the hemodialysis service is a risk, considering that multi-resistant bacteria are circulating. The major goal of MLVA is to determine if isolates are epidemiologically linked and followed over a relatively short period of time to see if they are related or unrelated. In this study, MLVA was helpful for discarding outbreaks in a hospital setting. MLVA is useful, fast, easy to perform, and particularly cheaper to the PFGE method already used in
PCR-based assays. ${ }^{23,24}$ In respect to other variables that were analyzed in patients, the highest frequency of rhinitis may be related to the fundamental role of eosinophils in the production of itching and predisposition to allergic reactions. ${ }^{25}$ Additionally, the nasal carriage of $\mathrm{S}$. aureus produces an immunomodulatory effect that could contribute to airway inflammation and allergic response in patients with allergic rhinitis. ${ }^{26}$ In the case of patients undergoing hemodialysis, presenting symptoms or signs may be overlapped by uremic pruritus. ${ }^{27}$ Study limitations were the low number of samples included as it was difficult to extrapolate results to all patients undergoing hemodialysis. $\mathrm{Ne}$ vertheless, as a pilot study this generated the first set of data on the characterization of $\mathrm{S}$. aureus from hemodialysis patients in Paraguay. As a perspective, we are interested in extending the study to other centers throughout the country.

The spread of $\mathrm{S}$. aureus can be controlled through reinforcement of appropriate use of antibiotics, hand washing and laboratory surveillance for $\mathrm{S}$. aureus, particularly in the nosocomial wards, in order to identify sources of outbreaks. The application of MLVA could help us to elucidate if isolates are epidemiologically linked, useful information for confirming or discarding outbreaks in the hospital environment. ${ }^{28,29}$

Figure 1. Genetic Relationship Observed by MLVA among Staphylococcus aureus Isolates

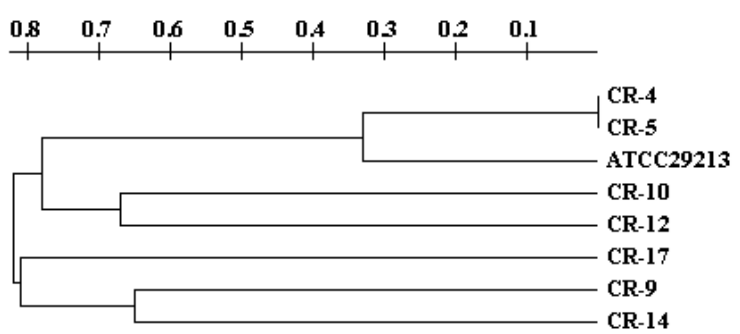

Legend: Dendrogram obtained with the software TreeCon v1.3b. Seven offourteen isolates were processed by the MLVA technique for cluster analysis including a control ATCC $(29213)$. The dendrogram shows the relationship between isolates, the abscissa numbers are indicative of the percentage of genetic variability. For example, between isolates CR-4 and CR- 5 there is no variability, but between CR- 4 and CR-17 there is $80 \%$ genetic variability. 


\section{References}

1. Giarola LB, dos Santos RR, Tognim MCB, Borelli SD, Bedendo J. Carriage frequency, phenotypic and genotypic characteristics of Staphylococcus aureus isolated from dialysis and kidney transplant patients at a hospital in Northern Paraná. Braz J Microbiol. 2012 Jul;43(3):923-30.

2. Greiner W, Rasch A, Köhler D, Salzberger B, Fätkenheuer G, Leidig M. Clinical outcome and costs of nosocomial and community-acquired Staphylococcus aureus bloodstream infection in haemodialysis patients. Clin Microbiol Infect. 2007 Mar;13(3):264-8.

3. Enright MC, Day NPJ, Davies CE, Peacock SJ, Spratt BG. Multilocus Sequence Typing for Characterization of Methicillin-Resistant and Methicillin-Susceptible Clones of Staphylococcus aureus. J Clin Microbiol. 2000 Mar;38(3):1008-15. 4. Saxena AK, Panhotra BR. The Prevalence of Nasal Carriage of Staphylococcus aureus and Associated Vascular Access-Related Septicemia Among Patients on Hemodialysis in Al-Hasa Region of Saudi Arabia. Saudi J Kidney Dis Transpl. 2003 Jan;14(1):30-8.

5. Sanavi S, Chods A, Afshar R. Catheter associated infections in hemodialysis patients. Saudi J Kidney Dis Transpl. 2007 Jan;18(1):43.

6. Vandecasteele SJ, Boelaert JR, De Vriese AS. Staphylococcus aureus Infections in Hemodialysis: What a Nephrologist Should Know. Clin J Am Soc Nephrol. 2009 Aug;4(8):1388-400.

7. Schmid H, Romanos A, Schiffl H, Lederer SR. Persistent nasal methiciIlin-resistant Staphylococcus aureus carriage in hemodialysis outpatients: a predictor of worse outcome. BMC Nephrol. 2013 Apr; 14:93.

8. Lai CF, Liao CH, Pai MF, Chu FY, Hsu SP, Chen HY, et al. Nasal carriage of methicillin-resistant Staphylococcus aureus is associated with higher all-cause mortality in hemodialysis patients. Clin J Am Soc Nephrol. 2011 Jan;6(1):16774 .

9. Peacock SJ, de Silva CD, Justice A, Cowland A, Moore CE, Winearls CG, et al. Comparison of Multilocus Sequence Typing and Pulsed-Field Gel Electrophoresis as Tools for Typing Staphylococcus aureus Isolates in a Microepidemiological Setting. J Clin Microbiol. 2002 0ct;40(10):3764-70.

10. Chung S, Yi J, Jang MH, Joo S-I, Ra EK, Kim SY, et al. Comparison of Modified Multiple-locus Variable-number Tandem-repeat Fingerprinting with Pulsed-field Gel Electrophoresis for Typing Clinical Isolates of Staphylococcus aureus. Ann Lab Med. 2012 Jan;32(1):50-6.

11. Manfredi EA, Leotta GA, Rivas M. Multiplex PCR for the detection of sea, seb, sec, sed and see genes of Staphylococcus aureus. Characterization of isolates from food. Rev Argent Microbiol. 2010 Sep;42(3):212-5.

12. Sabat A, Krzyszton-Russjan J, Strzalka W, Filipek R, Kosowska K, Hryniewicz W, et al. New Method for Typing Staphylococcus aureus Strains: Multiple-Locus Variable-Number Tandem Repeat Analysis of Polymorphism and Cenetic Relationships of Clinical Isolates. J Clin Microbiol. 2003 Apr;41(4):1801-4. 13. Sambrook J, Russell D. Molecular Cloning. A Laboratory Manual. 3rd ed. New York: Cold Spring Harbor Laboratory; 2001.

14. Jamil B, Bokhari MT, Saeed A, Mukhtar Bokhari MZ, Hussain Z, Khalid T, et al. Bacteremia: Prevalence and antimicrobial resistance profiling in chronic kidney diseases and renal transplant patients. J Pak Med Assoc. 2016 Jun;66(6):705-9.
15. Mohajeri P, Azizkhani S, Farahani A, Norozi B. Cenotyping of coa and aroA Cenes of Methicillin-Resistant Staphylococcus aureus Strains Isolated From Nasal Samples in Western Iran. Jundishapur J Microbiol. 2016 Jan;9(1):e26460. 16. Verhoeven PO, Gagnaire J, Haddar CH, Grattard F, Thibaudin D, Afiani A, et al. Identifying Hemodialysis Patients With the Highest Risk of Staphylococcus aureus Endogenous Infection Through a Simple Nasal Sampling Algorithm. Medicine (Baltimore). 2016 Apr;95(14):e3231.

17. Price A, Sarween N, Gupta I, Baharani J. Meticillin-resistant Staphylococcus aureus and meticillin-susceptible Staphylococcus aureus screening in a cohort of haemodialysis patients: carriage, demographics and outcomes. J Hosp Infect. 2015 May;90(1):22-7.

18. Devraj A, Siva Tez Pinnamaneni V, Biswal M, Ramachandran R, Jha V. Extranasal Staphylococcus aureus colonization predisposes to bloodstream infections in patients on hemodialysis with noncuffed internal jugular vein catheters. Hemodial Int. 2016 Jun; doi:10.1111/hdi.12450.

19. Zacharioudakis IM, Zervou FN, Ziakas PD, Mylonakis E. Meta-Analysis of Methicillin-Resistant Staphylococcus aureus Colonization and Risk of Infection in Dialysis Patients. J Am Soc Nephrol. 2014 Sep; 25(9):2131-41.

20. Lim CJ, Cheng AC, Kennon J, Spelman D, Hale D, Melican G, et al. Prevalence of multidrug-resistant organisms and risk factors for carriage in long-term care facilities: a nested case-control study. J Antimicrob Chemother. 2014 Jul;69(7):1972-80.

21. Rabelo MA, Bezerra Neto AM, Loibman So, Lima JL, Ferreira EL, Leal NC, et al. The occurrence and dissemination of methicillin and vancomycin-resistant Staphylococcus in samples from patients and health professionals of a university hospital in Recife, State of Pernambuco, Brazil. Rev Soc Bras Med Trop. 2014 Aug;47(4):437-46.

22. Reyes J, Rincón S, Díaz L, Panesso D, Contreras GA, Zurita J, et al. Dissemination of Methicillin-Resistant Staphylococcus aureus USA300 Sequence Type 8 Lineage in Latin America. Clin Infect Dis. 2009 Dec;49(12):1861-7.

23. Tenover FC, Vaughn RR, McDougal LK, Fosheim GE, McGowan JE Jr. Multiple-Locus Variable-Number Tandem-Repeat Assay Analysis of Methicillin-Resistant Staphylococcus aureus Strains. J Clin Microbiol. 2007 Jul;45(7):2215-9. 24. Malachowa N, Sabat A, Gniadkowski M, Krzyszton-Russjan J, Empel J, Miedzobrodzki J, et al. Comparison of Multiple-Locus Variable-Number Tandem-Repeat Analysis with Pulsed-Field Gel Electrophoresis, spa Typing, and Multilocus Sequence Typing for Clonal Characterization of Staphylococcus aureus Isolates. J Clin Microbiol. 2005 Jul;43(7):3095-100.

25. Garnacho-Montero J, Huici-Moreno MJ, Gutiérrez-Pizarraya A, López I, Márquez-Vácaro JA, Macher $\mathrm{H}$, et al. Prognostic and diagnostic value of eosinopenia, C-reactive protein, procalcitonin, and circulating cell-free DNA in critically ill patients admitted with suspicion of sepsis. Crit Care. 2014 Jun;18(3):R116. 26. Gröger M, Bernt A, Wolf M, Mack B, Pfrogner E, Becker S, et al. Eosinophils and mast cells: a comparison of nasal mucosa histology and cytology to markers in nasal discharge in patients with chronic sino-nasal diseases. Eur Arch Otorhinolaryngol. 2013 Sep;270(10):2667-76.

27. Çevik C, Yula E, Yengil E, Gülmez M , Akbay E. Identification of nasal bacterial flora profile and carriage rates of methicillin-resistant Staphylococcus aureus in patients with allergic rhinitis. Eur Arch Otorhinolaryngol. 2014 Jan;271(1):103-7. 


\section{Original Article}

28. Mettang T, Kremer AE. Uremic pruritus. Kidney Int. 2015 Apr;87(4):685-91. 29. Kim YC, Kim MH, Song JE, Ahn JY, Oh DH, Kweon OM, et al. Trend of methicillin-resistant Staphylococcus aureus (MRSA) bacteremia in an institution with a high rate of MRSA after the reinforcement of antibiotic stewardship and hand hygiene. Am J Infect Control. 2013 May;41(5):e39-43.

\section{Acknowledgments}

Special thanks to Dr. Marcos Martinez and the staff of the Nephrology Department of Hospital de Clínicas (Asuncion, Paraguay) for their support at the interviews with the patients and the kindly provided permission to access the clinical data.

Conflict of Interest Statement it Funding

None of the authors shows any conflicts of interest during the investigation or publication of this article.

Author Contributions

Conception and design the work/idea: RASI, CRCV, GAVR, JP, RMGF. Collect data/obtaining results: RASI, CRCV, GAVR. Analysis and interpretation of data: RASI, FR, RMGF. Write the manuscript: RASI. Critical revision of the manuscript: FR, E. Approval of the final version: RASI, FR, CRCV, GAVR, JP, RMGF. Contribution of patients or study material: CRCV, GAVR, JP. Administrative or technical advice: FR, JP, RMGF..

Cite as:

Silvero Isidre RA, Rodríguez Acosta F, Cristaldo Vargas CR, Velázquez Romero GA, Plans Perrota JF, Guillén Fretes RM. Molecular Characterization of Staphylococcus aureus Isolates Obtained from Hemodialyzed Patients at the Hospital de Clínicas of Paraguay: A pilot study. Int J Med Students. 2017 Jan-Apr;5(1):1419. 